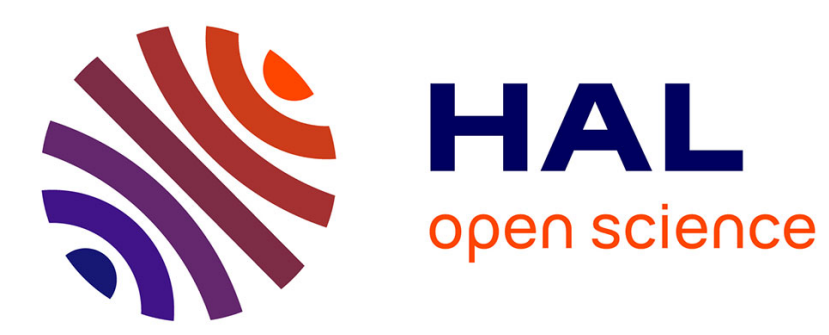

\title{
Héraclès/Jésus dans la sophistique impériale
}

Michel Tardieu

\section{To cite this version:}

Michel Tardieu. Héraclès/Jésus dans la sophistique impériale. Archimède: archéologie et histoire ancienne, 2019, 6, pp.48-54. halshs-02894255

\section{HAL Id: halshs-02894255 \\ https://shs.hal.science/halshs-02894255}

Submitted on 8 Jul 2020

HAL is a multi-disciplinary open access archive for the deposit and dissemination of scientific research documents, whether they are published or not. The documents may come from teaching and research institutions in France or abroad, or from public or private research centers.
L'archive ouverte pluridisciplinaire HAL, est destinée au dépôt et à la diffusion de documents scientifiques de niveau recherche, publiés ou non, émanant des établissements d'enseignement et de recherche français ou étrangers, des laboratoires publics ou privés. 


\section{ARCHIMĖDE $N^{\circ} \mathbf{6}$

DOSSIER THÉMATIQUE :

HISTOIRES DE FIGURES CONSTRUITES : LES FONDATEURS DE RELIGION

1 Guillaume DUCCEUR

Introduction

9 Philippe SWENNEN

Zarathushtra, une construction liturgique

16 Kyong-Kon KIM

Laozi. De la figure du maître mythique à la divinité taoïque

29 Guillaume DUCCEUR

Buddha et la marche sur les eaux : du prodige à la condition de l'arhant

39 Jean-Marie HUSSER

Le martyre de Jean-Baptiste, préfiguration de la Passion du Christ ?

48 Michel TARDIEU

Héraclès/Jésus dans la sophistique impériale

55 Anne-Sylvie BOISLIVEAU

Mahomet entre exégèse, hagiographie, rite et histoire

71 DOSSIER THÉMATIQUE :

JOUER DANS L'ANTIQUITÉ : IDENTITÉ ET MULTICULTURALITÉ GAMES AND PLAY IN ANTIQUITY: IDENTITY AND MULTICULTURALITY

213 VARIA 


\title{
HÉRACLĖS/JÉSUS DANS LA SOPHISTIQUE IMPÉRIALE
}

\author{
Michel TARDIEU \\ Professeur honoraire \\ Collège de France \\ mtardieu@wanadoo.fr
}

\section{RÉSUMÉ}

Les controverses mutuelles entre chrétiens et non-chrétiens ont été nombreuses au cours des premiers siècles de l'ère chrétienne et durant toute l'Antiquité tardive. La présente contribution se propose donc de revenir sur I'histoire de la construction de la figure d'Héraclès telle qu'elle fut forgée par l'empereur Julien dans sa dia-

MOTS-CLÉS

Héraclès,

Jésus,

Julien,

Héracleios,

Apollonios,

Philostrate,

Évangiles,

biographie,

sophistique,

néoplatonisme. tribe antichrétienne. À la vieille mythologie relative aux exploits héroïques d'Héraclès est ainsi substituée une trame narrative qui reprend les grandes étapes de la biographie traditionnelle de Jésus développée par les auteurs des Évangiles.
The mutual controversies between Christians and non-Christians were numerous during the first centuries of the Christian era and throughout the late Antiquity. This paper proposes to reassess the history of the construction of the figure of Heracles as it was forged by the emperor Julian in his antichristian diatribe. To the old mythology in relation to the heroic exploits of Heracles is thus substituted a narrative plot that takes up the main stages of the traditional biography of Jesus developed by the authors of the Gospels.

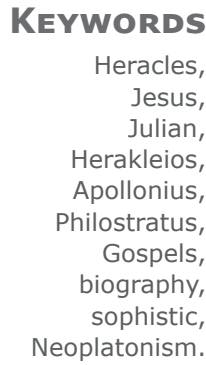




\section{MODALITÉS DES ÉQUIVALENCES}

Les historiens modernes des religions, à la suite de la littérature ancienne et médiévale, nous ont habitués à ce que Jésus soit mis en parallèle avec telle ou telle figure mythologique, grecque ou autre. Les traditions et récits au point de départ des rapprochements concernent des divinités à mystères, restituées par les rituels d'initiations. Le mode essentiel d'équivalence pour la divinité qui sert de terme de comparaison consiste à ressusciter après une mise à mort violente. Ceci s'applique pour le domaine égyptien à Osiris et, pour le monde grec et panhellénique, à Dionysos [1]. Du côté de I'Asie occidentale, des rapprochements analogues ont été effectués à propos du dieu syrien Adonis, du phrygien Attis, de I'iranien Mithra [2]. Depuis I'Antiquité, I'argumentation des équivalences oscille entre des extrêmes. Les polémistes antichrétiens s'évertuent à montrer que la représentation chrétienne de Jésus reproduit maladroitement un modèle antérieur de divin, en conséquence de quoi la filiation divine prêtée à Jésus est à leurs yeux infondée [3]. Pour les auteurs chrétiens, en revanche, la comparaison a pour but de mettre en évidence I'attente de Jésus fils de Dieu à travers des figures majeures de la fiction poétique [4], ce que le grand ouvrage fondateur de l'apologétique chrétienne appelle «préparation évangélique [5] ». Au début

[1] De la bibliographie pléthorique sur ce type de comparatisme, retenons pour Osiris : BONNEL TOBIN 1985 CANNUYER 1997. En ce qui concerne Dionysos, I'analyse de WILLERS 1992 a le mérite de faire le lien avec la mythologie nonnienne.

[2] De la lassante bibliographie sur Mithra/Jésus, il n'y a guère à retenir que l'imagerie concernant l'épisode de la visite des mages à Bethléem (MASTROCINQUE 2017). Pour Adonis et Attis, voir note 6.

[3] Ainsi chez Celse cité par Origène, Contre Celse, I 70. Un raisonnement similaire amène le théologien chrétien dissident, Marcion, à dénier chez Jésus toute possibilité de filiation divine par rapport au dieu des Juifs, le rédempteur étant nécessairement étranger et supérieur à un démiurge ; sur ce débat: HARNACK 2003, p. 140-143. du $X^{e}$ S., une étude quasi-exhaustive de la triade Osiris-Adonis-Attis ouvrit la voie à des recherches comparées élargies selon d'autres modalités [6].

Les reconstructions négatives ou positives de la personnalité de Jésus par de la mythologie ne portent pas seulement sur des divinités organisées à l'intérieur de panthéons, mais aussi sur des humains divinisés ayant leur cycle propre de traditions, tels Orphée, Héraclès, Pythagore. Le parallélisme Orphée/Jésus, inauguré du côté chrétien par Clément d'Alexandrie ( $\mathrm{II}^{\mathrm{e}} \mathrm{s}$.), a pour fondement la même thématique de mort/résurrection, qui avait présidé au rapprochement entre Dionysos et Jésus [7]. Avec Pythagore, dont à la fin de l'Antiquité les néoplatoniciens multiplient les Vies pour se donner l'illusion d'avoir à leur disposition une alternative crédible à Jésus, on assiste à un élargissement de la modalité par du narratif biographique : ce n'est plus seulement l'apothéose finale du héros qui sert d'élément de comparaison, mais son profil de faiseur de prodiges dans le cadre d'une biographie générale recomposée [8]. Une tradition de provenance néoplatonicienne va jusqu'à assimiler purement et simplement Pythagore à Jésus, en attribuant au premier les origines qui caractérisent le second selon les récits bibliques. En effet, dans la recension du commentaire de Proclus aux

[4] Par exemple, Actéon dévoré par ses propres chiens (Ovide, Mét., III 138-252), cf. POSSAMAI-PEREZ 2004.

[5] Eusèbe de Césarée, Préparation Évangélique, 9 vol. (Sources Chrétiennes, Cerf). Cette apologie a été composée dans la deuxième décennie du $\mathrm{IV}^{\mathrm{e}} \mathrm{s}$.

[6] FRAZER 1906. La pertinence des équivalences est discutée par ZELLER 1991.

[7] Sur ce point : JoURDAN 2006. L'Orphée musicien est, par sa spécialité, interculturel et interreligieux. Orphée/ David : STERN 1970 ; Orphée/Christ : FRIEDMAN1999.

[8] Vie recomposée la plupart du temps à partir de sommaires biographiques propres aux contes de tradition orale, ainsi que le montre DUNDES 1977. 
Vers $d$ 'or pythagoriciens, transmise en arabe par le savant nestorien du $\mathrm{xI}^{\mathrm{e}}$ S., Ibn al-Ṭyyib [9], la biographie de Pythagore proclame dès le début la filiation divine du philosophe : «Pythagore était de I'île de Samos, et on dit que la prophétie (al-kahāna) annonça sa naissance et qu'il est né d'une vierge ( $\min$ 'adhrā') [10] ». Le reste de la biographie est conforme au portrait traditionnel de Pythagore dans la philosophie grecque [11]. Il y a débat pour décider si ce commentaire attribué à Proclus est réellement de ce scholarque ou de quelque autre néoplatonicien, par exemple Hiéroclès [12]. Il n'est pas mentionné, en tout cas, dans la liste des ouvrages d'Ibn al-Tayyib compilée par Ibn Abī Ușaybīa [13]. Pour ce qui est de la tradition faisant naître Pythagore d'une vierge à l'instar du Christ, il s'agit, à mon avis, d'un transfert à l'épisode de la naissance de Pythagore de ce que I'on racontait à propos de la fille du philosophe. Restée vierge, celle-ci serait devenue maîtresse d'un chœur de vierges dont elle aurait réglé le genre de vie sur «les doctrines de la chasteté » (castitatis doctrinis), autrement dit selon un type monastique chrétien. Jérôme de Stridon ( $\operatorname{IV}^{\mathrm{e}} \mathrm{s}$.) met à profit cette tradition dans un inventaire érudit de pratiques ascétiques gréco-romaines ayant, selon lui, quelque rapport avec les dispositions des naissances virginales extraordinaires attribuées tant au Bouddha qu'à Jésus [14]. En réalité, Jérôme amplifie selon ses propres choix de vie un épisode biographique de I'histoire de Mya, fille de Pythagore, conduisant le chœur des jeunes filles de Crotone, épisode qu'il a lu dans la Vie de Pythagore de Porphyre (§ 4).

Héraclès est aussi, mais en plus complexe, un autre cas d'humain divinisé. Né de mère humaine, il possède comme Dionysos la filiation divine puisqu'il est fils de Zeus. Mais, à la différence des autres équivalences qui supposent les subtils cheminements des initiations et des mystères, il est atteignable directement. Il est le type même du héros populaire, facile à imaginer, compatissant et secourable. L'originalité du rapprochement avec Jésus tient au fait que le mode de comparaison ne dépend plus désormais d'un épisode unique terminal de la biographie du héros grec, en l'occurrence mise à mort suivie d'une apothéose, mais qu'il se fonde sur la totalité de ce qu'on peut appeler l'« histoire d'une vie ». Ce cas d'espèce a été I'objet en 1955 d'une étude novatrice de la part de Marcel Simon dans un petit livre (205 p.), chefd'œuvre de subtilité et d'érudition maîtrisée [15]. La figure de l'Héraclès chrétien s'y déploie au terme de I'idéalisation progressive du héros dans la tradition grecque, depuis Euripide et Prodicos jusqu'aux cyniques (ils ont Héraclès pour patron) puis chez les allégoristes stoïciens où il est assimilé au Logos. Dans les analyses en apparence paradoxales de Marcel Simon, l'image de Jésus est peu à peu digérée et refondée par celle de I'Héraclès philosophique, marquant par là une étape décisive vers la sortie du conflit culturel propre aux débuts du christianisme [16]. Cette prise de position concrétise le renouvellement de perspective et de méthode intervenu dans I'histoire universitaire strasbourgeoise de I'histoire des religions, d'Alfaric à Simon, du prédécesseur au successeur [17], du représentant de la thèse mythologique des « origines chrétiennes ». par l'exégèse historico-critique (Jésus en construction historicisée
[9] Abū I-Faraj 'Abd Allāh Ibn al-Ṭayib, mort en 1043 à Bagdad, philosophe, médecin et exégète. Sur cette personnalité et son œuvre : GRAF 1947, p. 160-162. Le nom et les dates d'Ibn al-Ṭayyib sont discutés par FAULTLESS 2003, p. 177-178. Un état récent des recherches sur Ibn al-Ṭayyib (domaines philosophique et médical exceptés) est fourni par FAULTLESS 2010.

[10] Ibn at-Ṭayib, Proclus' Commentary on the Pythagorean Golden Verses, ed. LINLEY 1984, p. 2, 5-6. La comparaison des filiations divines en contexte égyptien est étudiée par BRUNNER-TRAUT 1960 et 1988.

[11] Le seul disciple éminent de Pythagore, qui soit nommé (LINLEY 1984, p. 4, 11), est «Empédocle » (sic!), du moins selon la lecture que Neil Linley donne de la graphie arabe du manuscrit. À mon avis, il faut lire, non pas Empédocle, mais Jamblique.

[12] Sur ce point, voir O'MEARA 1989, p. 231-232 (apprécie ce commentaire « as a further evidence of the influence of Iamblichus' revival of Pythagoreanism »).

[13] Ibn Abī Ușaybī'a, 'Uyūn al-anbā' fĩ țabaqāt al-ațibbā, ed. Nizār Rị̣ā, Beyrouth, 1965, p. 325.
[14] Jérôme de Stridon, Adversus Iovinianum, I 42. Sur le genre littéraire et la visée polémique de ces inventaires de naissances extraordinaires, voir TARDIEU 2006.

[15] SIMON 1955. L'Auteur (1907-1986), professeur d'histoire des religions à l'Université de Strasbourg depuis 1947 et doyen de la Faculté des Lettres (1948-1968), fonda dans cette même faculté le Centre de recherches d'histoire des religions de Strasbourg (1954) ; I'année suivante paraissait Hercule et le christianisme. Parmi les comptes rendus substantiels, provenant de bords opposés, mais unanimes dans l'éloge : MOREAU 1956, MARROU 1956. Réception du livre chez les doctorants: DANVOYE 2002, BOUCHARD 2008.

[16] SimON 1973 revient sur cet aspect des choses en élargissant les conclusions de ROSE 1938, non prises en compte dans SIMON 1955.

[17] Prosper Alfaric (1876-1955) est le premier titulaire de la chaire d'histoire des religions de 1919 à 1945. Marcel Simon lui succède en 1947 (supra, n. 15). 
de la croyance chrétienne) au comparatiste des contextes philosophiques et littéraires qui ont produit l'enrichissement réciproque d'Héraclès et de Jésus en tant que figures de l'intercession.

\section{LA LIMITATION DE LA TOUTE-PUISSANCE}

Dans une allocution prononcée le 18 juin 1970 à la Faculté des Lettres et Sciences humaines de I'Université de Strasbourg, Henri-Irénée Marrou soulignait que l'auteur d'Hercule et le christianisme était « capable de communier à la fois avec les derniers païens, qui ont essayé de trouver dans Héraclès un contre-Christ, comme avec les chrétiens cultivés de [I']Antiquité tardive [18]». Quels retournements de pensée ont-ils joué pour aboutir à ce contre-Christ dont parle Marrou ? Un élément important dans l'enjeu du débat à l'arrière-plan de la dialectique de I'apport mutuel chère à Marcel Simon est la notion de toute-puissance envisagée dans des applications qui en limitent nécessairement la portée. La mise en œuvre de cet objectif couvre l'ensemble de la sophistique impériale en sa pluralité interprétative, d'Apollonius de Tyane à l'empereur Julien. Le moment fort du débat a été certainement I'interprétation à donner au « miracle de la pluie ». qui eut lieu en 174 lors de la campagne de Marc-Aurèle contre les Quadi, peuple germanique établi au nord de la frontière danubienne (actuelle Tchéquie). L'épisode est l'objet de maintes publications [19]. L'armée romaine privée d'eau et harcelée par l'ennemi court à la défaite. Mais un orage vient opportunément à son secours. On connaît tous les points de vue sur I'événement. À la question de savoir : qu'est-ce qui a fait pleuvoir ce jour-là ?, la réponse constamment signifiée par-delà la multiplicité des opinions est la suivante : C'est ce(lui) qui a la puissance sur les choses. Autrement dit, selon la diversité des interprétations, I'Arnouphis des mages égyptiens, le Julianus des théurges chaldéens, I'Hermès/Mercure/ Thot des monnaies romaines, le Jésus des chrétiens, I'Empereur de I'historiographie officielle. Les bas-reliefs de la Colonne Aurélienne sur la Piazza Colonna (Rome) représentent I'artisan démiurgique du miracle en vieillard chevelu et barbu, aux épaules puissantes et aux bras étendus, d'où ruisselle sur les soldats la pluie bienfaisante : tel est le Notus « aux ailes humides » (madidis alis) décrit par Ovide dans ses vers sur le déluge originel [20].

Pour la pluralité interprétative, ce n'est plus Zeus/ Jupiter qui fait pleuvoir, mais la parole de l'Empereur, les incantations d'Égyptiens ou de Chaldéens, les prières des chrétiens. Dans l'ordre hiérarchique du monde, la toute-puissance divine s'est déplacée vers la puissance des noms magiques, intercesseurs capables d'opérer des médiations à la demande. Mais le philosophe Marc-Aurèle lui-même écarte cette possibilité : «Ou bien les dieux sont impuissants ou bien ils sont puissants. Alors, s'ils sont puissants, pourquoi ne les pries-tu pas pour qu'ils t'accordent de ne pas redouter telle ou telle chose, plutôt que de les prier pour que telle ou telle chose t'arrive ou ne t'arrive pas [21] ? ». La pluie ressortit à ce qui arrive, elle est du tí gínetai qui ne dépend ni de nous ni de la divinité. Les pouvoirs divins sont limités et César ne peut pas tout, critique philosophique qui ne touche pas seulement les écrivains classiques de l'époque impériale ou les chrétiens gnostiques refusant d'accorder la toute-puissance au dieu biblique, mais qui rejaillit aussi sur la perception que I'on pouvait avoir des prodiges attribués à des figures aussi populaires qu'Héraclès. Les miracles de Jésus mis en avant par la catéchèse chrétienne primitive semblaient, en définitive, plus proches et concrets que les travaux d'Héraclès dont s'occupaient les poètes et les allégoristes. Face aux progrès de la nouvelle croyance, l'empereur Julien n'hésita pas à attribuer au héros grec le miracle de Jésus marchant sur les eaux du lac de Tibériade : « Je suis persuadé, écrit-il, qu'il (Héraclès) marchait sur la mer comme s'il se fût agi de la terre ferme [22] ». Héraclès redevint une alternative crédible pour les païens par imitation de Jésus. Les biographies évangéliques de Jésus permettaient de recomposer le héros populaire en sauveur du monde. Cette représentation d'Héraclès en substitut de Jésus reste avant tout polémique. Son but est de montrer que la culture traditionnelle du pouvoir en place, autrement dit I'hellénisme païen, possède une figure identique à celle de Jésus et véhiculant une symbolique similaire. Du point de vue de I'empereur Julien, cela signifiait que devenir chrétien était inutile, puisqu'il existait la même chose dans le paganisme. Le texte suivant déploie avec un art consommé la sophistique de l'empereur.

[18] MARROU 1970, p. 21.

[19] En dernière analyse ISRAEOLOWICH 2008 (avec bibliographie).

[20] Ovide, Métamorphoses, I, 164-169.

[21] Marc-Aurèle, Écrits pour soi-même, IX 40, texte et traduction dans CHAPOT-LAUROT 2001, p. 392.

[22] Julien, Contre Héracleios le Cynique, 14, 219D4-5, édition-traduction ROCHEFORT 1963, p. 63. La référence au miracle évangélique a été repérée par BIDEZ 1930, p. 253 et 397 ; SIMON 1955, p. 144 et 162. Elle n'est pas signalée dans ROCHEFORT 1963. 


\section{LA RÉAPPROPRIATION ANTICHRÉTIENNE D'HÉRACLÈS}

L'extrait, qui provient du discours de Julien Contre Héracleios le Cynique, est un texte continu et non un centon de phrases disparates. Cherchant à dépoussiérer Héraclès de sa mythologie grecque invraisemblable et à faire de lui un modèle biographique, Julien reproduit à la lettre les épisodes narratifs qui servent de cadre à la vie de Jésus dans la littérature chrétienne primitive. Chaque phrase tirée des Synoptiques et du quatrième évangile s'applique à Héraclès. La toute-puissance du nom de Jésus, autrement dit ses miracles, modèle désormais I'histoire d'Héraclès.

« Héraclès fut, dit-on, petit enfant, et son corps divin se développa peu à peu : on raconte qu'il fréquenta des maîtres. Il fit des campagnes, dit-on, et il triompha partout, mais son corps connut aussi la fatigue. Tout cela lui arriva, mais à une échelle plus qu'humaine : ainsi, dans les langes, son étranglement des démons, puis son combat contre les éléments mêmes de la nature - la chaleur et le froid -, ensuite sa lutte avec les situations les plus insurmontables et les plus irréductibles - je veux dire le manque de nourriture et la solitude - , et aussi, je crois, sa traversée de la mer elle-même sur une coupe d'or - à laquelle je dénie, les Dieux m'en sont témoins, toute existence réelle en tant que "coupe", mais je suis persuadé qu'il marchait sur la mer comme s'il se fût agi de la terre ferme. Qu'y avait-il d'insurmontable pour Héraclès ? Qui n'eût pas obéi à son corps divin et très pur, les éléments nommés ci-dessus étant soumis à la puissance démiurgique et efficace de son esprit impollu et pur ? C'est lui que le grand Zeus engendra pour être, avec I'aide d'Athéna Pronoia, le sauveur du monde : il l'avait placé sous la tutelle de cette déesse tout entière émanée de son être total. Il le rappela ensuite vers lui par le feu de la foudre - signal divin de la lumière éthérée par lequel il ordonnait à son fils de le rejoindre. Puisse du moins, sur ce sujet, Héraclès nous être favorable, à moi et à vous! ». [23]

Le corps divin d'Héraclès se développe à l'imitation de celui de Jésus : « L'enfant grandissait et se fortifiait, tout rempli de sagesse ». (LC 2, 40). Les campagnes et triomphes d'Héraclès sont vus à travers les épisodes évangéliques racontant les succès populaires de Jésus : foules de Galilée accourues pour l'entendre (LC 5, 15) ; entrée triomphale à Jérusalem (Mt 21, 1-11; Mc 11, 1-11 ; Lc 19, 28-40; Jn 12, 12-19). Comme Jésus à Gethsémani et durant la passion, Héraclès connaît la fatigue et la souffrance. L'attaque d'Héraclès dans ses langes par les démons sert de contrepoids à l'épisode évangélique racontant qu'Hérode, les grands prêtres et les scribes ont cherché à tuer Jésus lors de la visite des mages, mais que Jésus leur échappa par la fuite en Égypte (Lc 2, 12-15). On peut penser également à d'autres épisodes : Jésus enfant assis dans le Temple au milieu des docteurs de la loi (Lc 2, 41-52); Jésus enseignant à la synagogue de Capharnaüm (LC 4, 31-32). L'efficacité antidémoniaque des langes de Jésus est mentionnée par un apocryphe chrétien, la Vie de Jésus en arabe (ch. 10-11), dans un contexte de polémique anti-idolâtrique : le fils d'un prêtre égyptien païen ayant utilisé un lange de Jésus comme tarbouche, les spectateurs voient les démons s'enfuir de son corps comme des corbeaux ou des serpents [24]. Le combat d'Héraclès contre les éléments, le chaud et le froid, est une allusion aux miracles de Jésus (LC 4, 33-5, 14) dans la perspective d'une explication rationaliste des guérisons. Le manque de nourriture et la solitude d'Héraclès servent de répliques aux situations extrêmes rapportées dans la biographie évangélique de Jésus : tentation au désert (LC 4, 1-13), Jésus retiré dans les déserts, (LC 5,16). La coupe remise à Héraclès par le dieu Hélios lors de l'enlèvement des bœufs de Géryon dans l'île d'Érythie est un mythe, à l'instar de l'épisode évangélique de Jésus marchant sur les eaux (Mt 14, 25-26; Mc 6, 48-49; Jn 6, 19). Le père d'Héraclès-Jésus est appelé « le grand Zeus »., dénomination du dieu des Juifs dans les papyrus magiques et la littérature classique, c'est aussi le dieu Père des Oracles chaldaïques; Athéna Providence est la dénomination païenne du SaintEsprit, présent lors du baptême de Jésus (Mt 3, 13-17; Mc 1, 9-11). Le signal divin par lequel le grand Zeus ordonne à son fils de le rejoindre assimile la mort d'Héraclès à celle de Jésus sur la croix (Mt 27, 45-54; Mc 15, 33-39; Lc 23, 44-48 ; Jn 19, 28-30) selon une conception docète.

Le modèle sophistique de Julien dans la construction de ce contre-Christ a été Philostrate de Lemnos.

[23] Julien, Contre Héracleios le Cynique, 14, 219C220A, traduction ROCHEFORT 1963, p. 63-64, modifiée. Les notes de ce texte dans la Collection des Universités de France ne mentionnent aucune référence évangélique. Cet oubli a été heureusement réparé par BoufFARTIGUE 1992, p. 166-168, qui écrit : «Les Évangiles se posent en modèle contraignant [de Julien] ».

[24] Je remercie Guillaume Dye de m'avoir signalé ce texte. 
Né vers 165, celui-ci meurt sous le règne de Philippe I'Arabe (244-249). Philostrate est un familier du pouvoir impérial romain. Il rédige la Vie d'Apollonius de Tyana à la demande de l'impératrice Julia Domna, épouse de Septime Sévère (reg 193-211) [25]. Julia Domna est une princesse syrienne, née vers 170, fille d'un prêtre païen d'Émèse (Hims) ; en 187, elle épouse Septime Sévère, dont elle a un fils I'année suivante, Bassianus, le futur empereur Caracalla ; en 193, Septime Sévère devient empereur et meurt en 211. Son fils Caracalla lui succède. Il est assassiné en 217 près de Harrān, Julia meurt peu de temps après.

En 1832, la Vie d'Apollonius fut l'objet par Ferdinand Christian Baur d'une monographie phare de I'histoire comparée des religions [26]. Le théoricien de l'École hégélienne de Tübingen a écrit là probablement son meilleur livre. En des pages très denses, il y démontre que le roman, construit sur ce qui faisait le succès de la littérature évangélique (intrication des discours et des prodiges), traduit une sorte d'hommage rendu par le paganisme à une religion qui le supplantait déjà. L'historien Eduard Meyer alla en sens contraire. « En dépit de contacts occasionnels », reconnaissait-il, « il n'y a pas le moindre indice que Philostrate ait voulu décrire en Apollonios un parallèle païen au Christ ou, d'une façon générale, qu'il se soit soucié d'une manière quelconque du christianisme [27] ». Les travaux d'aujourd'hui sur la question oscillent entre les deux points de vue [28]. Les miracles racontés dans la Vie d'Apollonius et dont Carmen Padilla restitue la morphologie par les récits de contes oraux [29] montrent à l'œuvre le mécanisme complexe des échanges dont s'est nourri l'empereur Julien. En constatant chez Philostrate I'emploi qui y était fait des miracles du Christ recomposés en prodiges à la gloire d'un pythagoricien itinérant, l'empereur apprit comment il lui fallait à son tour protéger I'hellénisme et contribuer par là à la stabilité de son pouvoir impérial.

[25] Ainsi qu'il le précise lui-même au début de la Vie d'Apollonius : «Comme je faisais partie du cercle de l'impératrice - car elle admirait et aimait tous les propos relatifs à l'éloquence - elle me donna mission de récrire ces mémoires et de pourvoir à leur publication. » (Vie d'Apollonius de Tyane, I 3).

[26] BAUR 1832, p. 226-227. Dans la ligne de Baur, en moins original : RÉVILLE 1865. Ce dernier, pasteur de l'église wallone de Rotterdam, rentre en France en 1880 , alors que Jules Ferry vient de créer la première chaire d'histoire comparée des religions au Collège de France ; Réville y est élu avec I'appui de Renan et de Gambetta.

[27] MEYER 1917, p. 161, n. 3.

[28] BOWIE 1978, DZIELSKA 1986, RoBiANO 1989, VAN UYTFANGHE 2009, PRAET 2017.

[29] PADILLA 1991.

\section{BIBLIOGRAPHIE}

BAUR, Ferdinand Christian, 1832, « Apollonius von Tyana und Christus, oder das Verhältnis des Pythagoräismus zum Christenthum. Ein Beitrag zur Religionsgeschichte der ersten Jahrhunderte nach Christus »., repris dans Id., Drei Abhandlungen zur Geschichte der alten Philosophie und ihres Verhältnisses zum Christentum, hrsg. von Eduard ZeLLER, Leipzig, Fues's Verlag, 1876, p. 1-227.

BIDEZ, Joseph, 1930, La vie de l'empereur Julien, Paris, Les Belles Lettres.

Bonnel, Roland G. \& Tobin, Vincent A., 1985, «Christ and Osiris. A Comparative Study »., in S. I. Groll (ed.), Pharaonic Egypt. The Bible and Christianity, Jerusalem, Magnes Press, p. 1-29.

Bouchard, Isabelle, 2008, Hercule à la défense du christianisme, Québec, Université Laval (Internet).

BowIE, Ewen Lyall, 1978, « Apollonius of Tyane : Tradition and Reality »., in Aufstieg und Niedergang der römischen Welt, II/16/2, Berlin, De Gruyter, p. 1652-1699.

Bouffartigue, Jean, 1992, L'Empereur Julien et la culture de son temps, Paris, Études Augustiniennes.

BRUNNER-TrAUT, Emma, 1960, « Geburtsgeschichte der Evangelien im Lichte ägyptologischer Forschungen »., Zeitschrift für Religions- und Geistesgeschichte, 12, p. 97-111. 
Brunner-Traut, Emma, 1988, «Pharao und Jesus als Söhne Gottes »., dans Id., Gelebte Mythen : Beiträge zum altägyptischen Mythos, Darmstadt, p. 31-59.

Cannuyer, Christian, 1997, « Osiris et Jésus, les bons pélicans »., dans Fr. Boespflug, Fr. Dunand (dir.), Le comparatisme en histoire des religions, Paris, Cerf, p. 223-238.

СhAPOT, Frédéric, \& LAUROT, Bernard, 2001, Corpus de prières grecques et romaines, Turnhout, Brepols.

DanvoYe, Stéphanie, 2002, « Hercule et le christianisme : autour des imaginaires mythiques »., Folia Electronica Classica, 4, http://bcs.fltr.ucl.ac.be/FE/04/danvoye.html

Dundes, Alan, 1977, The Hero Pattern and the Life of Jesus, repris dans R. A. Segal (ed.), In Quest of the Hero, Princeton, Princeton University Press, 1990, p. 179-223.

DzielskA, Maria, 1986, Apollonius of Tyana in Legend and History, Translated by Piotr Pienkowski, Roma, "L'Herma" du Bretschneider.

FAULtLess, Julian, 2003, « The two recensions of the Prologue to John in Ibn al-Tayyib's Commentary on the Gospels »., in David Thomas (ed.), Christians at the Heart of Islamic Rule. Church Life and Scholarship in 'Abbasid Iraq, Leiden, Brill.

FAultless, Julian, 2010, «Ibn al-Ṭayyib»., dans D. Thomas, A. Mallett (eds.), Christian Muslim Relations. A Bibliographical History, Vol. 2 (900-1050), Leiden Brill, p. 667-697.

FrAZER, James G., 1906, Adonis, Attis, Osiris. Studies in the History of Oriental Religion, London.

FrIEDMAN, John B., 1999, Orphée au Moyen Âge, Fribourg, Éditions Universitaires.

GRAF, Georg, 1947, Geschichte der christlichen arabischen Literatur, Studi e Testi 133, Vol. II, Città del Vaticano.

HARNACK, Adolf von, 2003, [édition allemande 1924], Marcion. L'évangile du Dieu étranger. Une monographie sur I'histoire de la fondation de I'Église catholique, Traduit par B. Lauret et suivi de contributions de B. Lauret, G. Monnot et É. Poulat. Avec un essai de M. Tardieu, « Marcion depuis Harnack »., Paris, Cerf.

IsRAELOWICH, Ido, 2008, « The Rain Miracle of Marcus Aurelius : (Re-)construction of Consensus »., Greece and Rome, 55, p. 83-103.

Jourdan, Fabienne, 2006, « Dionysos dans le Protreptique de Clément d'Alexandrie »., Revue de I'histoire des religions, 223, p. 265-282.

LINLeY, Neil, 1984, Ibn at-Tayyib. Proclus' Commentary on the Pythagorean Golden Verses, Arabic Text and Translation, New York, State University of New York at Buffalo.

MarRou, Henri-Irénée, 1956, «Compte rendu de M. Simon, Hercule et le christianisme »., Revue d'études anciennes, 58, p. 418-421.

MARrou, Henri-Irénée, 1970, « Allocution»., dans Hommage au doyen Marcel Simon de la part de ses amis et de ses élèves, Strasbourg, Imprimerie Régionale, p. 19-21.

Mastrocinque, Attilio, 2017, The Mysteries of Mithra, Tübingen, Mohr Siebeck, p. 134-136 (Orientalische Religionen in der Antike, 24).

MeYer, Eduard, 1917, «Apollonios von Tyana und die Biographie des Philostratos »., repris dans Id., Kleine Schriften, t. II, Halle (Saale), Max Niemeyer, 1924.

Moreau, Jacques, 1956, « Compte rendu de M. Simon, Hercule et le christianisme »., L'Antiquité classique, 25, p. 539-543 O'meARA, Dominic, 1989, « The Arabic Commentaries on the Golden Verses Attributed to Iamblichus and Proclus »., dans Id., Pythagoras Revived. Mathematics and Philosophy in Late Philosophy, Oxford, Clarendon Press.

Padilla, Carmen, 1991, Los milagros de la Vida de Apolonio de Tiana : morfología del relato de milagro y géneros afines, Córdoba, Ediciones El Amendro.

Possamai-Perez, Marylene, 2004, « Comment Actéon devint le Christ »., dans P. Nobel (dir.), Textes et cultures, I, Besançon, Presses Universitaires de Franche-Comté, p. 187-210.

Praet, Danny, 2017, «Inclusivité et exclusivité dans la Vie d'Apollonius de Tyane »., Revue de l'histoire des religions, 234, p. 661-688.

RÉviLLe, Albert, 1865, «Le Christ païen du IIIe siècle : Apollonius de Tyane et la cour des Sévères »., Revue des Deux Mondes, 59, p. 620-654.

Robiano, Patrick, 1989, « Apollonius de Tyane »., Dictionnaire des philosophes antiques, t. I, Paris, CNRS Éditions, p. 289-294.

ROCHefORT, Gabriel, 1963, L'Empereur Julien. Fuvres complètes, Tome II/1 ${ }^{\mathrm{èr}}$ partie, Discours de Julien Empereur, Paris, Les Belles Lettres (Collection des Universités de France).

Rose, Herbert Jennings, 1938, « Herakles and the Gospels »., Harvard Theological Review, p. 113-142.

Srmon, Marcel, 1955, Hercule et le christianisme, Strasbourg, Publications de la Faculté des Lettres de I'Université de Strasbourg.

Simon, Marcel, 1973, «Early Christianity and Pagan Thought. Confluences and Conflicts »., Religious Studies, 9, p. $385-399$. STERN, Henri, 1970, « Un nouvel Orphée-David dans une mosaïque du VI siècle »., Académie des Inscriptions et BellesLettres. Comptes rendus des séances, 114, p. 63-79.

TARDIEU, Michel, 2006, « Nativités païennes : les treize "royaumes" de l'Apocalypse gnostique d'Adam »., dans B. Feichtinger, St. Lake, H. Seng (hrsg.), Körper und Seele. Aspekte spätantiker Anthropologie, München, Leipzig, Saur, p. 9-65. Van Uytranghe, Marc, 2009, «La Vie d'Apollonius de Tyane et le discours hagiographique »., dans Kr. Demoen, D. Praet (eds.), Theios Sophistes. Essays on Flavius Philostratus' Vita Apollonii, Leiden, Brill, p. 335-374.

Willers, Dietrich, 1992, «Dionysos und Christus »., Museum Helveticum, 49, p. 141-151.

ZELLER, Dieter, 1998, « Hellenistische Vorgaben für den Glauben an die Auferstehung Jesu ? », dans R. Hoppe, U. Busse (hrsg.), Von Jesus zum Christus. Christologische Studien. Festgabe für Paul Hoffmann, Berlin, De Gruyter, p. 71-91. 